\section{Personality disorder}

(these categories ARE NOT mutually exclusive):

1. Sociopathy including criminalty, explosive/aggressive behaviour. sexual perversion and addiction.

2. Schizoid personality disorder.

3. Hysterical personality disorder.

4. Obsessional personality disorder.

5. Passive dependent personality disorder.

6. Addiction not amounting to sociopathy.

7. Sexual deviation not amounting to sociopathy

8. None.

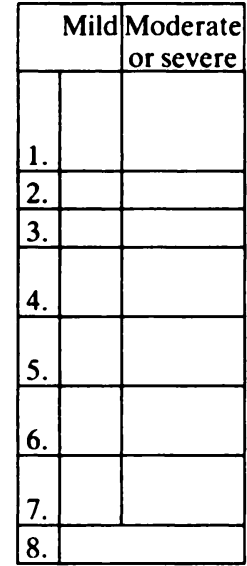

III.Schizoid personality (not necessarily amounting to personality disorder), and whether or not associated with psychiatric illness, other personality disorder, mental retardation or organic impairment.)

1. Definitely present

2. Doubtfully present

3. Absent

IV.Mental Handicap:

1. Severe

2. Mild

3. Absent

V. Organic cerebral impairment:

1. Definitely present

2. Doubtfully present

3. Absent

Western General Hospital,

Edinburgh EH4 $2 \mathrm{XU}$

\section{MISINTERPRETING ROLE CHANGES AS CHANGES OF PERSON}

DeAR SIR,

Dr MacCallum (Journal, June 1984, 144, 649-650) describes patients who exhibit "a syndrome of misinterpreting role changes as changes of person". In his article, Dr MacCallum likens this syndrome to the syndromes of delusional misidentification and concludes by offering a tentative dynamic explanation of the phenomena: "perhaps the separation of the other roles was an expression of the mothers' resentment of these new aspects in the relationship, and the forced dependence on their daughters". There are some features of the histories which are at odds with $\mathrm{Dr}$ MacCallum's comparisons and conclusions.
Firstly, two of the patients (Mrs E. E. D. and Mrs E. C.) identify their daughters correctly when they are present. Thus, Mrs E. E. D. asks 'Violet' (her daughter) to "get the other Violet" and Mrs E. C. calls her daughter Emma (a nurse) for a confidential chat and then comments on, "that other woman . . . you know, the nurse". In a similar fashion when the third patient, Mrs H. M. is talking to Dr MacCallum about the people who look after her, she names "the person who gets me up" and 'Margaret', (her daughter). It seems, therefore, that all three patients are aware of the existence of their daughters and two of them recognise their daughters when they are present, making no attempt to dispute their identity. This is qualitatively different from the syndromes of delusional misidentification because in these, the identity of a person is consistently denied, even when they are present.

Secondly, Dr MacCallum maintains that there is no evidence of prosopagnosia even though he used the conversation of an "almost blind" woman who perserverates to exclude this possibility. Similarly, he tells us that the patients' sensoria are clear despite their cerebro-vascular disease. Both these statements are unjustified considering the sensitivity of the cognitive tests employed. It is more likely that their sensoria are frosted, or at least show evidence of some cognitive deficit.

Although many features of the patients' phenomenology are not presented, there is another hypothesis that may better account for the details in the report. This hypothesis stems from information processing and communication theories. According to these, information is processed in two types of code; the digital and the analogue, terms frequently used in computing and mathematics. Spoken language is one of the more complex examples of a digital code. whereas non-verbal communication (kinesics), represent an analogue transcription. Relationships are predominantly represented in an analogue form, but some degree of transcription to the digital code can occur. It is the digital linguistic code that leads to abstract thought and the classification of identity (Watzlawick, Beavin \& Jackson 1968).

Identity can be classified in many ways. Three of the more important classes are; name, (Violet, Emma, etc.); kinship, (mother, father, etc.) and role. (cook, nurse, etc.). The classification of identity by kinship and role differs from classification by name. This is because kinship and role classes are abstracted from relationships, whereas the name class is not. For example, the identity of a cook depends on someone cooking for someone; similarly, the identity of a mother depends on the mother/infant relationship. This is not true for the identity by name. 
Dr MacCallum's patients have no difficulty coding identity into these classes in the majority of their relationships. They even identify their daughters correctly some of the time. However, their ability to transcribe the analogue relationship into the appropriate digital code, fails when they recall certain interactions with the daughter. As Dr MacCallum points out, the phenomenon of misidentification occurs in the setting of a role reversal.

The patients are all infirm and consequently depend on their daughters for care. When the daughter provides care, she adopts a mothering role. This change in relationship produces a paradox in the digital code, because the mothers experience their daughters as mothering them. To resolve this paradox of daughters as mothers, the patients recall and conceptualise the daughter as an 'other'. They signify the difference in relationship with the linguistic qualifier 'other'. Furthermore, the misidentified 'other' is given the same name as the daughter ("Violet, get the other Violet") because the classification of identity by name is not involved in the paradox.

In this way, the patients' inappropriate use of language resembles the way patients with mild dementia use language (Critchley 1970). Their thought patterns are similar to the thought patterns children exhibit in the concrete operational phase of cognitive development.

1. Watzlawick P., Beavin J. H. \& Jackson; D. D. Jackson (1968) The Pragmatics of Human Communication, London: Faber \& Faber.

2. Critchley MacDonald (1970) Aphasiology, London: Edward Arnold.

3. Piaget J. \& Inhelder B. (1969) The Psychology of the Child, London: Routledge \& Kegan Paul.

St. Georges Hospital,

P. F. Bolton

Blackshaw Road,

London SWI7

Dear Sir,

I was interested in the paper by $\mathrm{Dr}$ MacCallum (Journal, June 1984, 144, 649-650), on the misinterpreting of role changes as changes of person, from two points of view. At a personal level, I have in recent months witnessed the gradual dementia of my 90 -yearold mother who is now in nursing home care. This has been accompanied by an increasing tendency to confuse the roles of the significant men in her life. While correctly identifying and naming me as her son, she often refers to my sibs as our children, may reminisce as if I were her father and can confuse my son with me. This conflated male represents a more or less idealised version, much as Dr MacCallum's patients identified the real daughter with the caring role, and the confusion has been accompanied by a reduction in a lifelong tendency to be unreasonably disappointed in, or critical of, others. This would seem to represent a similar but reversed form of confusion to that described in the three women in the paper. Theoretically, it would seem that such confusions of roles and individuals might be seen as parallel to the infant's original inability to combine perceptions of different roles into a single complex image of an individual-the process described as splitting by object-relations theorists. The later-achieved integrations of these separate role perceptions are, presumably, as vulnerable to aging as are other aspects of memory and cognitive function.

(Name and address supplied)

\section{DEPRESSIVE SYMPTOMS IN PREGNANCY}

DEAR SiR,

The exhaustive study of Drs Kumar and Robson (Journal 144, 35-47) shows convincingly that psychiatric symptoms in pregnancy are common. There remains debate as to whether these symptoms are of importance in the prediction of postnatal depression, a condition of considerable clinical importance (Kumar and Robson; Playfair and Gowers, 1981). We have carried out a prospective survey of depressive symptomatology in a cohort of pregnant women in the setting of general practice.

All those women attending for antenatal care at a group practice during 1982 were selected for study. The women were screened for depressive symptoms at routine antenatal clinic visits. Screening was by means of a microcomputer-delivered Hamilton Depression Rating Scale (HDRS) modified for self rating (Carr et al, 1981; Margo et al, 1983). Depressive symptoms identified in this way were confirmed by clinical interview.

Of 134 women entering the study, 17 subsequently miscarried or had termination of pregnancy. In 117 women screened throughout pregnancy the prevalence of depressive symptoms increased, reaching a peak at 37-38 weeks. During weeks $8-12,24.3 \%$ scored more than 10 (outside the normal range for computer delivered HDRS, Carr et al, 1981) and 5.1\% scored more than 17 , in a range that would normally be consistent with requiring antidepressant therapy. At 25-28 weeks the prevalences were $25.5 \%$ scoring more than 10 , and $6.1 \%$ more than 17 . At $37-38$ weeks $45.7 \%$ scored more than 10 , and $11.8 \%$ more than 17 . Mean HDRS scores also rose throughout pregnancy, from 7.9 at 8-12 weeks to 10.3 at 37-38 weeks. 